\title{
ESTUDO DA APLICAÇÃO DA TÉCNICA DE SUPRESSÃO DE PÓ NAS EMISSÕES SECUNDÁRIAS DE MATERIAL PARTICULADO TOTAL DA PLANTA DE SINTERIZAÇÃO DA ARCELORMITTAL TUBARÃO*
}

\author{
Rafael Sartim ${ }^{1}$ \\ Thayná Vervloet Gomes ${ }^{2}$ \\ Bernardo Enne Corrêa da Silva ${ }^{3}$ \\ Tiago Agra Rosi ${ }^{4}$ \\ Flávio Busatto ${ }^{5}$ \\ Cláudio Marcos Dias Curvello Júnior ${ }^{6}$ \\ Priscilla da Cruz Cosmo ${ }^{7}$
}

\section{Resumo}

A indústria siderúrgica possui aspectos ambientais associados ao seu processo produtivo ligados em grande parte à emissão de material particulado, efluentes líquidos e geração de resíduos. A emissão de material particulado ocorre durante diversas etapas do processo produtivo, cabendo a estes a aplicação de sistemas para o controle ambiental. Em especial, a planta de sinterização possui como característica emissões primárias ligadas à exaustão da máquina, e secundárias referentes aos sistemas de captações em linhas de peneiramento, retorno de finos e pontos de transferência de correias transportadoras. Para o tratamento das emissões secundárias, a ArcelorMittal Tubarão possui um sistema de despoeiramento. Em virtude de melhorias de processo e alterações na produção, o sistema necessita de uma maior vazão para atendimento à demanda atual. Neste contexto, este trabalho se propôs a avaliar e testar a técnica de supressão de pó em pontos de transferência entre correias transportadoras para verificar sua aplicabilidade. Como conclusão, constatou-se que esta técnica é aplicável em regiões específicas com bons resultados na redução de emissão visível. Além disso, o uso do supressor reduz a quantidade de captações necessárias ao sistema complementando o controle do atual sistema de despoeiramento.

Palavras-chave: Sinterização; Supressor de pó; Material particulado; Controle ambiental.

\section{STUDY OF THE DUST SUPRESSION APPLICATION IN SECONDARY EMISSIONS OF PARTICULATE MATTER IN ARCELORMITTAL TUBARÃO SINTER PLANT}

\section{Abstract}

The steel industry has environmental aspects associated to the production process largely linked to the particulate matter emission, liquid effluents and waste generation. The emission of particulate matter is generated in various stages of the production processes that make necessary the application of air pollution control system. The sinter plant is characterized by the primary emissions which is associated to the offgas from the sister strand and secondary emissions which are generated mainly at the screening systems, sinter cooler and conveyor belt transfer chutes. In order to mitigate the secondary dust emissions ArcelorMittal Tubarão has a dedusting system. Due to process improvements and production plan modifications the system needs more flow rate in order to achieve the actual efficiency necessity. In this context, this study aimed to evaluate and test the dust suppression technique in transfer points, to check its applicability. It was concluded that this technique is applicable in specific with good results in the reduction of visible emission. Furthermore, the use of the dust suppressor reduces the amount of hoods and air flow necessary for the system.

Keywords: Sinter plant; Dust supression; Particulate matter; Environmental control.

${ }^{1}$ Engenheiro Mecânico, PhD, Especialista de Engenharia Mecânica, Área de Engenharia de Manutenção Mecânica / Departamento de Projetos e Engenharia, ArcelorMittal Tubarão, Serra, ES, Brasil.

2 Eng. Ambiental, Engenheira de Planejamento, Universidade Federal do Espírito Santo, Vitória, ES, Brasil.

3 Biólogo, Especialista de Meio Ambiente, Gerência de Meio Ambiente, ArcelorMittal Tubarão, Serra, ES, Brasil.

${ }^{4}$ Administrador, Supervisor de Manutenção, Área de Manutenção da Sinterização, ArcelorMittal Tubarão, Serra, ES, Brasil.

${ }^{5}$ Engenheiro Metalúrgico, Especialista de Controle Técnico de Processo da Sinterização, Área de Controle de Produção da Sinterização, ArcelorMittal Tubarão, Serra, ES, Brasil.

6 Técnico em Mecânica, Especialista de Aplicação de Eng. de Supressão, Depto. de Eng., Benetech, Serra, ES, Brasil.

7 Engenheira Ambiental, Analista Ambiental, Universidade Federal do Espírito Santo, Vitória, ES, Brasil. 


\section{INTRODUÇÃO}

Uma das etapas na produção do aço presentes em uma usina siderúrgica integrada consiste na aglomeração de minérios, cuja função é melhorar a permeabilidade da carga metálica. Existem quatro métodos de aglomeração de minérios: sinterização, pelotização, briquetagem e nodulização. O processo de aglomeração da sinterização possui finalidade de produzir o sínter, carga metálica utilizada para abastecer os Altos Fornos para produção de ferro gusa [1]. Além disso, a sinterização tem importante papel ambiental na reciclagem de resíduos gerados na unidade industrial, pois permite utilizar como matéria-prima do sínter materiais como pó do alto forno [2] e pó do precipitador eletrostático da própria sinterização [3], entre outros.

A emissão de material particulado é uma característica intrínseca ao processo de sinterização. As emissões primárias da sinterização são aquelas provenientes da operação da máquina de sínter, que funciona com a ignição do combustível presente na mistura e com o auxílio de pernas de vento para promover a condução dos gases de combustão provenientes da camada de sínter. Com isso, pode-se dizer que as emissões secundárias são todas as demais emissões relacionadas aos processos da planta de sinterização (resfriamento, peneiramento, transferências de material em correias transportadoras, dentre outros) [4].

$\mathrm{Na}$ sinterização, os pontos de transferência de material de uma correia transportadora para outra, denominados de chutes de transferência, são as áreas que merecem atenção no que diz respeito à questão ambiental associada ao manuseio de matérias-primas. Estes são pontos críticos de emissão fugitiva associada a esse tipo de transporte cuja melhor prática de controle é promover o uso de dispositivos de coleta e tratamento de material particulado (instalação de coifas de captação nas transferências, ligadas a um sistema de despoeiramento, geralmente um precipitador eletrostático ou filtro de mangas) [4-6].

No entanto, outra técnica para controlar as emissões de fontes extensas que pode complementar e/ou substituir o uso de um sistema de ventilação local exaustora, é o uso de sprays de água com ou sem produtos químicos. Este processo é chamado de supressão de pó, pois reduz as emissões nos pontos em que é aplicado, no entanto, não elimina a geração total do material particulado [7].

Os sprays de água, com ou sem agente químico, são utilizados em diversas aplicações, tais como: pilhas de matéria-prima, transferências entre correias transportadoras de material, mineração, umectação de vias, descarregamento de matérias-primas ou resíduos, entre outras. No entanto, a quantidade de material particulado emitida por essas atividades depende das características do material e da forma como é manuseado [8]. Além disso, a aplicabilidade do supressor de pó depende das características físicas do material (granulometria, umidade, forma) e do processo industrial aplicado a este [9].

Em muitos ambientes, o uso dos supressores é preferível. Os principais motivos são:

$\checkmark$ Eficiência;

$\checkmark$ Relação favorável de custo/benefício;

$\checkmark$ Rápida implementação;

$\checkmark$ Baixo custo de manutenção [10].

Os supressores atuam nas emissões atmosféricas em duas vertentes: supressão primária, que é aquela que evita a geração de emissão; e supressão secundária, que atua na supressão da poeira já emitida [11], que não devem ser confundidas com as emissões características do processo de sinterização. A atuação do 
supressor é exemplificada na Figura 1, em que a emissão primária está representada na região $\eta_{1}$ e a secundária na região $\eta_{2}$.

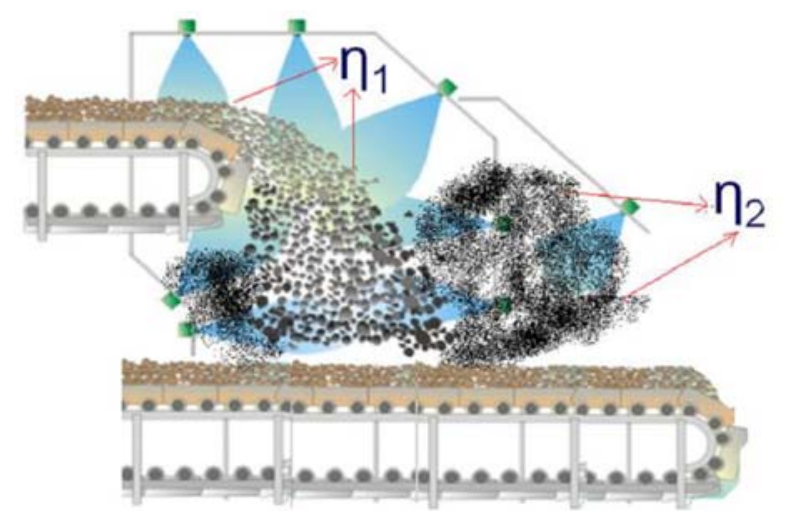

Figura 1. Efeitos da redução primária e secundária de emissão de pó pelo uso da supressão nas emissões fugitivas de uma transferência entre correias transportadoras [8].

Existem diversos fatores que influenciam na eficiência da supressão de pó, um deles é o uso de aditivos químicos, como os surfactantes, também chamados de tensoativos [8,12]. Os surfactantes são compostos químicos que apresentam uma porção hidrofílica e uma porção hidrofóbica, fazendo com que se posicionem preferencialmente nas interfaces entre fases fluidas com diferentes graus de polaridade (como nas interfaces óleo/água ou arlágua). Com isso, essas moléculas tem a capacidade de reduzir a tensão interfacial e/ou superficial, devido à formação de uma camada molecular ordenada na interface, formando uma micro-emulsão, onde os hidrocarbonetos podem solubilizar a água e vice-versa [12]. Esses compostos são utilizados como supressores de pó, pois reduzem a tensão superficial da água provocando os seguintes efeitos:

$\checkmark$ Redução do diâmetro da gota do spray;

$\checkmark$ Aumento no número de gotas para um dado volume de água; e

$\checkmark$ Diminuição do ângulo de contato, definido como o ângulo com o qual o líquido se encontra com a superfície do sólido [8].

O uso de surfactantes aumenta a razão em que as gotas são capazes de "molhar" o material particulado; assim, uma menor quantidade de água é utilizada na supressão para produzir os mesmos efeitos que uma aplicação comum, sem esse agente, otimizando o uso do recurso. Pequenas quantidades de surfactante podem ser injetadas no spray de água, tipicamente com uma diluição na faixa de 1:700 a $1: 1500$, o que melhora o umedecimento e, consequentemente, o controle da emissão de particulado [9].

No entanto, apesar de sua eficiência, os aditivos químicos como os surfactantes nem sempre são utilizados, devido a limitações como:

$\checkmark$ Surfactantes são significativamente mais caros do que a simples aplicação de água;

$\checkmark$ Podem alterar as propriedades do mineral ou material processado;

$\checkmark$ Podem danificar alguns equipamentos como correias transportadoras e vedações (na ausência de manutenção);

$\checkmark$ Os sistemas que usam surfactantes requerem maior conservação e manutenção do que o sistema típico, com água apenas [9].

A eficiência dos aditivos químicos depende de alguns itens, tais como:

$\checkmark$ Tipo de agente;

$\checkmark$ Natureza hidrofóbica das partículas; 
$\checkmark$ Tamanho das partículas de pó;

$\checkmark$ Concentração de pó;

$\checkmark \mathrm{pH}$ da água;

$\checkmark$ Minerais presentes na água utilizada [9].

As emissões secundárias de material particulado de uma planta de sinterização possuem características tais que permitem a aplicação ambos os sistemas de controle, seja por ventilação local exaustora ou pela técnica de supressão de pó [13]. Na planta de sinterização da AMT, há uma alta demanda de vazão para o Sistema de Despoeiramento Secundário. Atualmente, o sistema utiliza dois precipitadores eletrostáticos como equipamento de controle de poluição atmosférica, instalados em 1983 e reformados em 1997. No entanto, a partir do aumento de produção da empresa, ocorrido em 2007 (início da operação do alto forno $n^{\circ} 3$ ), a planta de sinterização da AMT sofreu algumas alterações, aumentando sua capacidade visando atender o novo plano de produção. Consequentemente, a demanda de vazão para o sistema despoeiramento aumentou gradativamente. Dessa forma, a aplicação de outra técnica complementarmente, como a supressão de pó, torna-se uma opção atrativa para melhorar a atual eficiência do sistema, visto os benefícios apontados.

Neste contexto, o objetivo do presente trabalho é estudar e testar a aplicação da técnica de supressão de pó em transferências entre correias transportadoras na planta da sinterização, para que possa complementar o sistema de despoeiramento existente, reduzindo a quantidade de coifas e dutos, o que contribui para a eficiência de captação nos demais pontos de emissão onde o sistema continuará atuando.

\section{MATERIAIS E MÉTODOS}

Foram realizados em junho de 2014 testes no sistema de despoeiramento secundário com o objetivo de avaliar preliminarmente e de forma visual o desempenho da técnica de supressão de pó, para assim verificar sua aplicação nas captações localizadas em chutes de transferência entre correias transportadoras.

Para o teste, foram instalados manifolds (dispositivo responsável por regular a distribuição de vazão) em alguns dos pontos passíveis de aplicação, com número de bicos, vazão e consumo do produto químico variando para cada local, conforme descrito na Tabela 1. No teste, foram avaliadas duas regiões:

$\checkmark$ Região do resfriador: onde a emissão avaliada ocorre após a descarga do resfriador, que despeja o sínter em um alimentador vibratório ligado à correia A, que com o auxílio de outras transportarão o material até a próxima etapa da fabricação do sínter, o peneiramento. Nessa região, foram instalados dois manifolds: um ao final da calha do alimentador vibratório e outro na correia A, conforme apresentado na Figura 2.

$\checkmark$ Região de envio do sínter para a área dos altos fornos, denominada Casa de Junção. Essa região se encontra após a Casa de Peneiramento, e o ponto avaliado refere-se à transferência do sínter entre as correias $\mathrm{B}$ e $\mathrm{C}$, local em que foi instalado um manifold, conforme apresentado na Figura 3.

Esses pontos de aplicação foram determinados levando em consideração principalmente a questão da umidade adicionada ao sínter, uma vez que este parâmetro influencia na qualidade deste material no processo metalúrgico de transformação do ferro gusa nos altos fornos. A técnica de supressão adiciona certa umidade ao material, conforme mostrado na Tabela 1, por isso, nem todos os pontos de emissão localizados em transferências de correias foram considerados viáveis. 
Além disso, levaram-se em conta as condições de processo, que impedem, por exemplo, a aplicação do produto na região de peneiramento em função da aderência do material às peneiras.

Tabela 1. Informações técnicas e locacionais referentes ao teste com o supressor de pó realizado na planta de sinterização da AMT

\begin{tabular}{c|c|c|c}
\cline { 2 - 4 } & \multicolumn{2}{c}{ Localização } \\
\cline { 2 - 4 } & $\begin{array}{c}\text { Alimentador Vibratório } \\
\text { para Correia A }\end{array}$ & Correia A & $\begin{array}{c}\text { Correia B para } \\
\text { Correia C }\end{array}$ \\
\hline $\begin{array}{c}\text { Capacidade do } \\
\text { Transportador (t/h) }\end{array}$ & 833 & 833 & 700 \\
\hline $\begin{array}{c}\text { Umidade Adicionada } \\
\text { Aproximada (\%) }\end{array}$ & $<0,3$ & $<0,3$ & $<0,5$ \\
\hline $\begin{array}{c}\text { Umidade Adicionada } \\
\text { Aproximada (L/h por } \\
\text { manifold) }\end{array}$ & 1388,5 & 555,4 & 3500 \\
\hline $\begin{array}{c}\text { Taxa de Diluição do } \\
\text { Agente }\end{array}$ & $1: 500$ a $1: 1000$ & $1: 500$ a $1: 1000$ & $1: 500$ a $1: 1000$ \\
\hline $\begin{array}{c}\text { Consumo do Agente } \\
\text { (L/h por manifold) }\end{array}$ & 1,39 a 2,78 & 0,56 a 1,11 & 3,5 a 7 \\
\hline $\begin{array}{c}\text { Configuração de } \\
\text { Aplicação }\end{array}$ & 1 manifold com 5 bicos & 2 manifolds com 2 & 1 manifold com 2 \\
bicos cada & bicos cada \\
\hline
\end{tabular}

$\checkmark$ Alimentador Vibratório: recebe o sinter após o material passar pelo resfriador e alimenta a correia A;

$\checkmark$ Correia A: transporta o sinter que será levado por outras correias à Casa de Peneiramento;

$\checkmark$ Correia B: transporta o sinter da Casa de Peneiramento para a Casa de Junção;

$\checkmark$ Correia C: localizada na Casa de Junção, também é responsável por transportar o sinter.

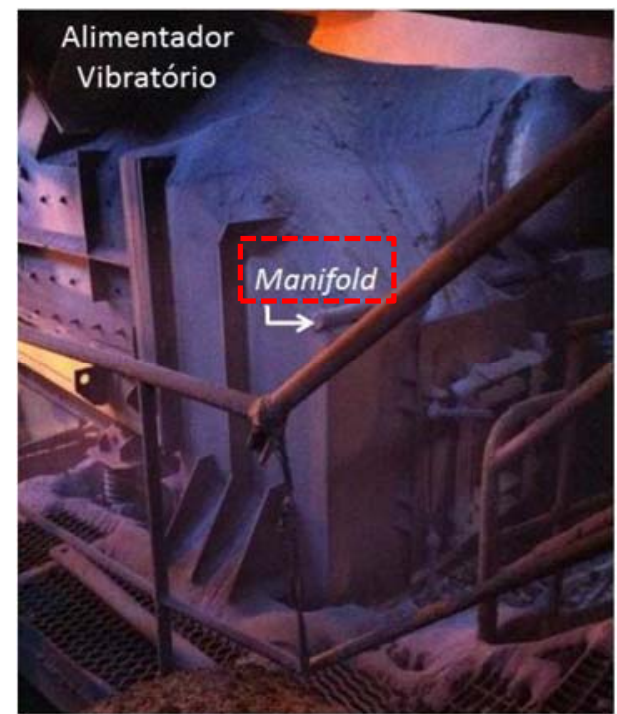

(a)

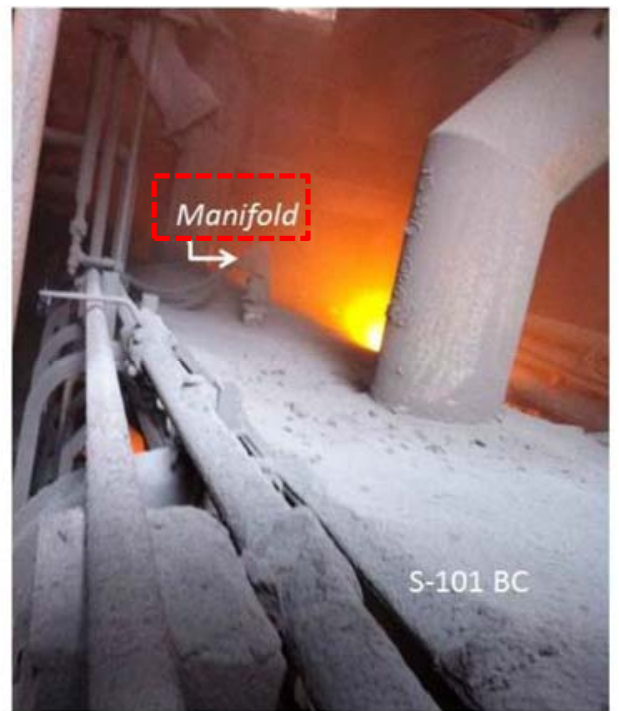

(b)

Figura 2. Supressor de pó na região do resfriador - (a) Alimentador Vibratório para à correia A; (b) correia A. 


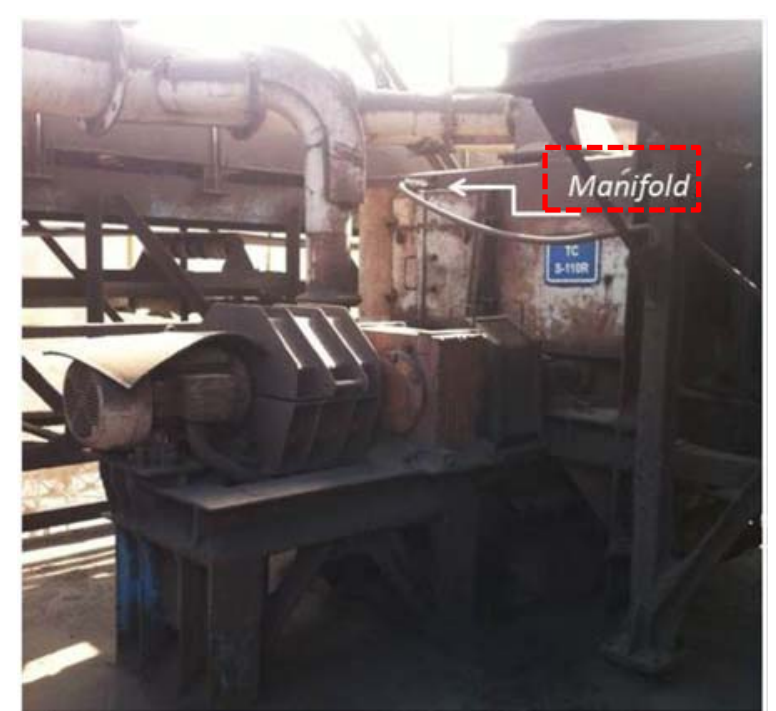

Figura 3. Supressor de pó na transferência de sinter da correia B para a correia C.

É importante mencionar que os testes descritos nesse trabalho foram realizados nos locais indicados na Tabela 1, no entanto, existem mais pontos onde sua aplicação é sugerida, e onde novos testes devem ser realizados.

Para testar a aplicação da tecnologia, foi feita a avaliação visual das emissões em duas situações distintas:

$\checkmark$ Sem atuação do sistema de despoeiramento secundário;

$\checkmark$ Com atuação apenas do supressor de pó.

A avaliação visual dessas condições permitiu observar a resposta do sistema à nova tecnologia, no caso da aplicação em transferências de correia.

Além disso, avaliou-se a umidade do material após aplicação do supressor, visando respeitar os limites aceitáveis para a produção nos altos fornos.

\section{RESULTADOS E DISCUSSÃO}

Conforme descrito no item 2, a técnica de supressão de pó foi aplicada nos seguintes pontos: região do resfriador e região de envio do sínter para a área dos altos fornos, denominada Casa de Junção.

Durante a realização dos testes, foi feita a avaliação visual da eficiência da técnica de supressão de pó. Foi observado que o uso da supressão de pó com agente químico como técnica de controle da poluição em transferências entre correias demonstrou boa eficiência. Notou-se, ainda, que quando o sistema de despoeiramento foi desativado e o sistema de supressão foi acionado, para que apenas este pudesse atuar, as emissões visíveis pontuais reduziram significativamente. Com isso, existe a possibilidade de aprofundamento nos estudos da substituição pontual de captações específicas em locais em que haja atuação do supressor, o que reduziria a demanda de vazão do sistema de despoeiramento secundário, uma das dificuldades atuais em plantas de sinterização.

As Figuras 4, 5 e 6 ilustram a situação da emissão nas condições de teste: sistema de supressão de pó desativado e sistema de supressão de pó em funcionamento. Destaca-se que em ambas as situações não há exaustão de ar nas captações, pois o sistema de despoeiramento secundário foi desligado durante os testes para que não interferisse na atuação da tecnologia testada.

Como é possível observar na Figura 4 (a), a emissão de material particulado durante a operação do alimentador vibratório é mais significante e facilmente notada 
visualmente. Com a aplicação do supressor, ilustrado pela Figura 4 (b), constata-se que o abatimento das partículas ocorre com boa eficiência, não sendo observado material particulado suspenso no ambiente ao redor.

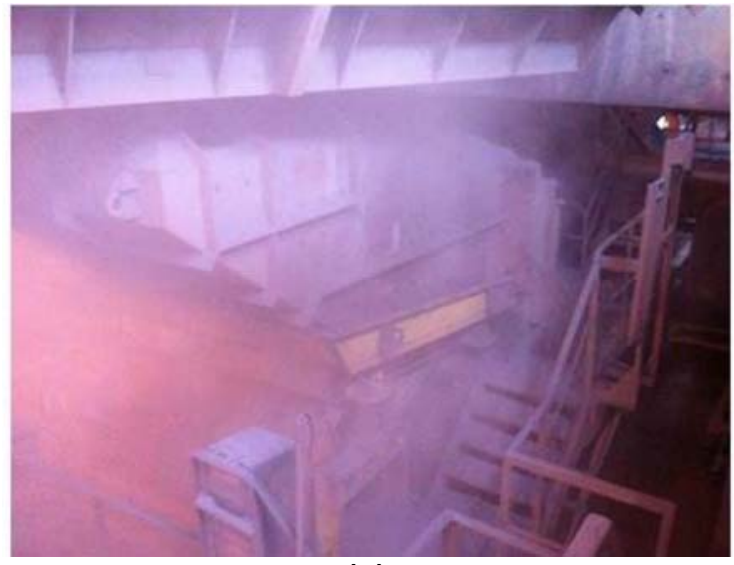

(a)

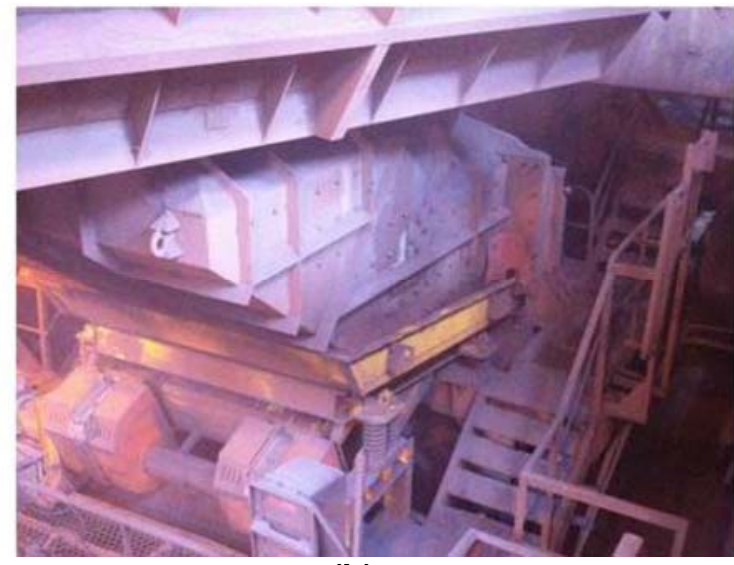

(b)

Figura 4. Região da descarga do Alimentador Vibratório para a correia $A$ - (a) Emissão visível quando o supressor de pó estava desativado; (b) Emissão visível reduzida em função da ação da tecnologia de supressão de pó.

Notou-se ainda que quando o supressor de pó foi utilizado, sua ação ainda pôde ser observada nas transferências de correia seguintes (quando estas não se encontravam muito distantes), como no caso em que o supressor é aplicado na correia $A$, surtindo efeito na transferência entre a própria correia $A$ para a correia $D$, e também entre a correia D para a correia E. Esta última situação (duas transferências após a aplicação do supressor) é apresentada na Figura 5.

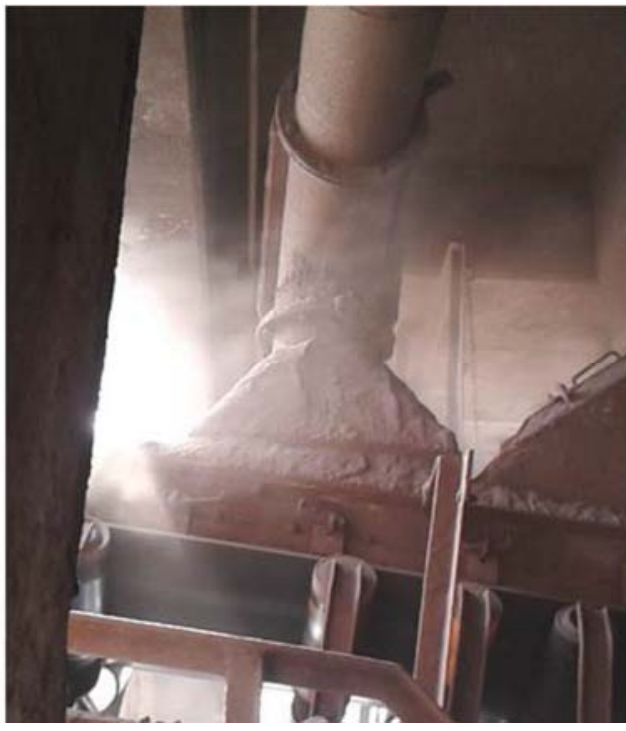

(a)

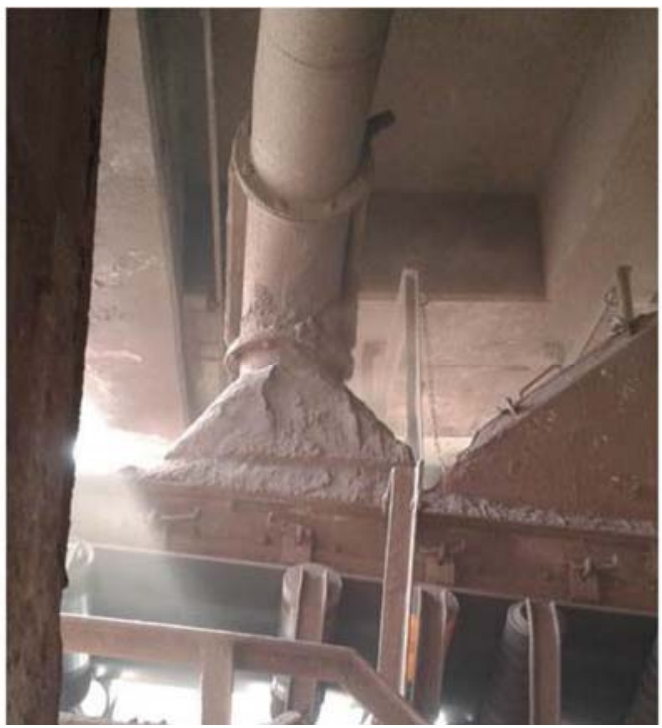

(b)

Figura 5. Região logo após o chute de transferência da correia $D$ para a correia $E$ - (a) Emissão visível quando o supressor de pó estava desativado; (b) Emissão visível reduzida em função da ação da tecnologia de supressão de pó.

$\mathrm{Na}$ outra região (Casa de Junção), apresentada na Figura 6, também se observa uma boa eficiência da supressão de pó na mitigação da emissão de material particulado em transferência entre correias. Na Figura 6 (b), não é visualizado 
material particulado suspenso na região após o chute de transferência, diferentemente do que se observa na Figura 6 (a).

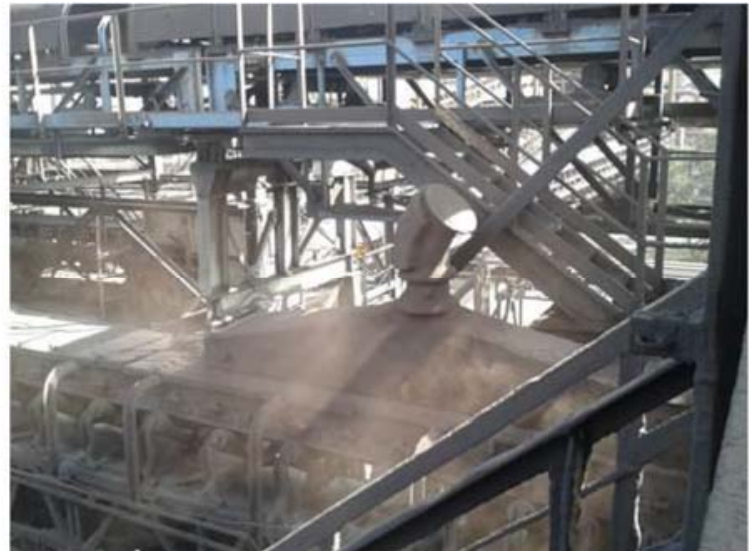

(a)

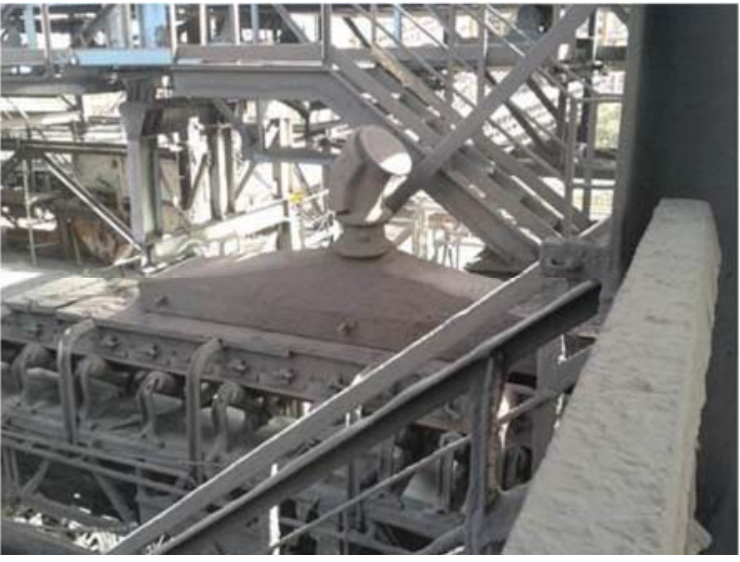

(b)

Figura 6. Região logo após o chute de transferência da correia B para a $C$ - (a) Emissão visível quando o supressor de pó estava desativado; (b) Emissão visível reduzida em função da ação da tecnologia de supressão de pó.

Além disso, foi retirada amostra de sínter após a aplicação do supressor a fim de verificar se a umidade do material é alterada de modo a afetar sua qualidade para os altos fornos. Os resultados dos testes de umidade se mostraram satisfatórios, uma vez que a média foi de $0,01 \%$. A umidade do sínter aceitável para atender a produção deve ser inferior $0,5 \%$.

\section{CONCLUSÃO}

O presente trabalho possui o objetivo de avaliar a aplicação da técnica de supressão de pó em transferências de correias transportadoras na sinterização, visando complementar o sistema existente. Após realização do teste com essa tecnologia na planta sinterização da AMT, verificou-se que sua utilização é aplicável para algumas transferências entre correias, devendo ser respeitado o processo produtivo e suas características, principalmente no que diz respeito à umidade adicionada ao sínter. Concluiu-se que com o uso do supressor de pó, é possível eliminar alguns pontos de captação, o que auxiliaria na melhoria das demais captações que permaneceriam sendo atendidas pelo despoeiramento secundário. Além disso, essa técnica pode ser utilizada também em caso de novos projetos ou projetos para aumento da capacidade do sistema, sendo o supressor uma alternativa para redução da vazão direcionada ao equipamento de controle, acarretando em redução no custo de implantação.

\section{Agradecimentos}

À ArcelorMittal Tubarão, pela oportunidade de desenvolver este trabalho. 


\section{REFERÊNCIAS}

1 ARAUJO, L. A. Manual de siderurgia. Editora: Arte \& Ciência, V.1, São Paulo - SP, 1997.

2 MACHADO, M. L. P. Siderurgia - da matéria-prima ao aço laminado. Centro Educacional de Educação Tecnológica do Espírito Santo - CEFET-ES - Gerência de Ensino Coordenadoria de Recursos Didáticos. Vitória, 2006.

3 RIBEIRO, M. M. Material particulado da planta de sinterização de usina siderúrgica integrada em composição de massa de cerâmica vermelha. 2010. 163 f. Tese (Doutorado em Engenharia e Ciências dos Materiais). Universidade Estadual do Norte Fluminense Darcy Ribeiro - UENF. [acesso em: 30 set. 2014]. Disponível em: http://uenf.br/posgraduacao/engenharia-de-materiais/wpcontent/uploads/sites/2/2013/07/Tese-Monica-M-Ribeiro-PDF.pdf.

4 JOINT RESEARCH CENTRE - European Commission. Best Available Techniques (BAT) Reference Document for Iron and Steel Production. Industrial Emissions Directive 2010/75/EU (Integrated Pollution Prevention and Control). 2013 [acesso em 26 fev. 2014]. Disponível em: http://eippcb.jrc.ec.europa.eu/reference/BREF/IS_Adopted_03_2012.pdf.

5 CHEN, X. L.; WHEELER, C. A.; DONOHUE, T. J.; MCLEAN̄, R.; ROBERTS, A. W. Evaluation of dust emissions from conveyor transfer chutes using experimental and CFD simulation. International Journal of Mineral Processing. 2012 [acesso em 20 jun. 2014]. Disponível em:

http://proceedings. asmedigitalcollection.asme. org/proceeding. aspx?articleid=2205634.

ULLMANN, A.; DAYAN, A. Exhaust volume model for dust emission control of belt conveyor transfer points. Powder Technology. 1998 [acesso em 08 abr. 2014]; 96(2): 139-147. Disponível em:

http://www.sciencedirect.com/science/article/pii/S0032591097033676.

7 SCHONBACH, B. H. Operational options for Dust Control. American Coal Council PRB Coal Use Seminar, Detroit MI, 2003 [acesso em 08 abr. 2014]. Disponível em: http://bhstechnical.com/techpapers/OpOptionsDustCtrl-BHS.pdf.

8 BLAZEK, C. F. The Role of Chemicals in Controlling Coal Dust Emissions. American Coal Council PRB Coal Use: Risk Management Strategies \& Tactics Course. 2003 [acesso em 08 abr. 2014]. Hyatt Regency Dearborn, Michigan. Disponível em: http://www.powerpastcoal.org/wp-content/uploads/2011/08/The-Role-of-Chemicals-inControlling-Coal-Dust-Emissions.pdf.

9 CECALA, A. B.; O'BRIEN, A.D.; SCHALL, J.; COLINET, J. F.; FOX, W. R.; FRANTA, R. J.; JOY, J.; REED, W. R.; REESER, P. W.; ROUNDS, J. R.; SCHULTZ, M. J. Dust Control Handbook for Industrial Minerals Mining and Processing. Department of Health and Human Services. Pittsburgh. 2012 [acesso em 08 abr. 2014]. Disponível em: http://www.cdc.gov/NIOSH/Mining/UserFiles/works/pdfs/2012-112.pdf.

10 SPRAYING SYSTEMS CO. Guide to Spray Technology for Dust Control. [acesso em 08 abr. 2014]. Disponível em:

http://www.spray.com/Literature_PDFs/B652_Dust_Control. pdf.

11 FASCHINGLEITNER, J.; HÖFLINGER, W. Evaluation of primary and secondary fugitive dust suppression methods using enclosed water spraying systems at bulk solids handling. Advanced Powder Technology 22. 2011 [acesso em 27 jun. 2014]; 236-244. Disponível em: http://www.sciencedirect.com/science/article/pii/S0921883110002554.

12 DESAI, J. D.; BANAT, I. M. Microbial production of surfactants and their commercial potential. Microbiology and Molecular Biology Reviews. 1997 [acesso em 08 abr. 2014]; 47-64. Disponível em: http://mmbr.asm.org/content/61/1/47.full.pdf.

13 ACGIH - American Conference of Governmental Industrial Hygienists. Industrial Ventilation: A Manual of Recommended Practice for Design. 27th edition. Cincinnati: 2010. 